where

and

$$
E^{i} \equiv a^{i} \xi+b^{i} \eta+d^{i} \lambda+e^{i}(\mu-\nu)
$$

$$
\Sigma a a^{\prime}=0, \quad \Sigma a a^{\prime \prime}=0, \quad \Sigma a^{\prime} a^{\prime \prime}=0 .
$$

Similarly, if $x=0, z=0$ be the second axis, we have

with

$$
E_{1}+\theta_{1} E_{1}{ }^{\prime}+f_{1} E_{1}^{\prime \prime}=0,
$$

$$
\Sigma a_{1} a_{1}{ }^{\prime}=0, \quad \Sigma a_{1} a_{1}{ }^{\prime \prime}=0, \quad \Sigma a_{1} a_{1}{ }^{\prime \prime}=0 .
$$

Moreover, every complex of the first system must be in involution with every complex of the second. These results show that the invariant of one of the complexes of each system vanishes. Since the discriminant of a series is invariant under linear transformation of the hexaspherical coördinates, surfaces having only plane lines of curvature can not be transformed into general surfaces having spherical lines of curvature by inversion in a linear complex. This configuration is treated in my dissertation, $\S 18$, III, $1 \beta$, page 45 . The cyclide becomes a quadrilateral inscribed in the absolute circle at infinity.

Cornele University,

April, 1904.

\title{
ADDITION TO A THEOREM DUE TO FROBENIUS.
}

BY PROFESSOR G. A. MILLER.

(Read before the San Francisco Section of the American Mathematical Society, April 30,1904.)

IN a paper presented by the writer at a recent meeting of the London mathematical society it is proved that the number of cyclic subgroups of order $p^{s}, p$ being any odd prime and $s>1$, which are contained in a non-cyclic group of order $p^{m}$ is always a multiple of $p$. From this theorem it follows directly that the number of cyclic subgroups of order $p^{s}$ in any group $G$ must be a multiple of $p$ whenever the Sylow subgroups of order $p^{m}$ are non-cyclic. When these Sylow subgroups are cyclic the number of the cyclic subgroups of order $p^{s}$ contained in $G$ is clearly of the form $1+\mathrm{kp}$. In all other cases $G$ contains $1+k p$ non-cyclic subgroups of order $p^{s}$. In other words, if $G$ 
contains one non-cyclic subgroup of order $p^{s}$ it must contain $1+k p$ non-cyclic subgroups of this order.

Frobenius has recently proved that the number of operators of $G$ which satisfy the equation $x^{n}=s_{1}$ is a multiple of $n$ whenever $s_{1}$ is an invariant operator of G.* Since a cyclic group of order $p^{s}$ has $p^{s-1}(p-1)$ distinct generators the results of the preceding paragraph prove that the number of operators of $G$ which satisfy the equation $x^{p s}=1$ without also satisfying the equation $x^{p s-1}=1$ is a multiple of $p^{s}$ whenever the Sylow subgroups of order $p^{m}$ in $G$ are non-cylic. In this case $G$ must contain a multiple of $p^{s^{\prime}+1}$ operators which satisfy the equation $x^{p s}=1,0<s^{\prime}<m$, since the number of its operators which satisfy this equation without also satisfying $x^{p^{s}-1}=1$, increased by those which satisfy the latter equation, is a multiple of $p^{s^{\prime}}$.

We proceed to prove that the number of operators of order $n=p^{s} m_{1}, m_{1}$ being prime to $p$, is always a multiple of $p^{s}$, whenever the Sylow subgroups of order $p^{m}$ in $G$ are non-cyclic. If. one of the prime factors $q$ of $n$ is such that $q-1$ is divisible by $p$ the theorem requires no proof since the number of the generators of a cyclic group of such an order is a multiple of $p^{s}$. When this condition is not satisfied it is only necessary to prove that some Sylow subgroup $P_{m}$ of order $p^{m}$ transforms just $l p$ cyclic subgroups of order $n$ into themselves.

If $P_{m}$ does not transform any cyclic subgroup of order $n$ into itself the theorem requires no proof. If it transforms such a subgroup into itself, all the operators of $P_{m}$ must be commutative with each of the operators of this subgroup whose order is prime to $p$. Hence $G$ contains the direct product of $P_{m}$ and the group generated by these operators. This direct product contains a multiple of $p$ cyclic subgroups of order $n$ since $P_{m}$ contains a multiple of $p$ cyclic subgroups of order $p^{s}$. If $G$ contains another cyclic subgroup of order $n$ which is transformed into itself by $P_{m}$, it must contain another such direct product, etc. As the cyclic subgroups of order $n$ in these direct products are distinct, the theorem is proved. Just as in the second paragraph, it may be observed that the number of operators of $G$ which satisfy $x^{n}=1$ is a multiple of $p^{s+1}, s<m$.

STANFORD UNIVERSITY, April, 1904.

* Frobenius, Berliner Sitzungsberichte, 1903, p. 895. 